\title{
Absorption-Modulated Crossed-Optical Fiber-Sensor Platform for Measurements in Liquid Environments and Flow Streams
}

\author{
Paul E. Henning and Peter Geissinger \\ Department of Chemistry and Biochemistry, University of Wisconsin-Milwaukee, 3210 North Cramer Street, \\ Milwaukee, WI 53211, USA \\ Correspondence should be addressed to Peter Geissinger; geissing@uwm.edu
}

Received 5 February 2017; Revised 19 April 2017; Accepted 7 May 2017; Published 13 June 2017

Academic Editor: Oleg Lupan

Copyright (C) 2017 Paul E. Henning and Peter Geissinger. This is an open access article distributed under the Creative Commons Attribution License, which permits unrestricted use, distribution, and reproduction in any medium, provided the original work is properly cited.

\begin{abstract}
A new evanescent-wave fiber sensor is described that utilizes absorption-modulated luminescence (AML) in combination with a crossed-fiber sensor platform. The luminescence signals of two crossed-fiber reference regions, placed on opposite sides of the stretch of fiber supporting the absorbance sensor, monitor the optical intensity in the fiber core. Evanescent absorption of the sensor reduces a portion of the excitation light and modulates the luminescence of the second reference region. The attenuation is determined from the luminescence intensity of both reference regions similar to the Beer-Lambert Law. The AML-Crossed-Fiber technique was demonstrated using the absorbance of the $\mathrm{Zn}(\mathrm{II})-\mathrm{PAN}_{2}$ complex at $555 \mathrm{~nm}$. A linear response was obtained over a zinc(II) concentration range of 0 to $20 \mu \mathrm{M}$ (approximately 0 to $1.3 \mathrm{ppm}$ ). A nonlinear response was observed at higher zinc(II) concentrations and was attributed to depletion of higher-order modes in the fiber. This was corroborated by the measured induced repopulation of these modes.
\end{abstract}

\section{Introduction}

Optical fiber sensors have been widely studied because they are inexpensive, flexible, compact, rugged, nonconductive, and immune to electromagnetic interference [1-4]. Also, these advantages permit remote sensing where sample collection is inconvenient, impractical, or impossible and remove sensitive equipment and personnel from harsh environments. One class of fiber sensors utilize the evanescent wave generated at the core-cladding interface when light undergoes total internal reflection. The evanescent wave is a standing wave that decays exponentially in the cladding normal to the core-cladding interface. An absorbing or scattering particle in the cladding within the range of the evanescent wave can extract optical energy from the fiber core. Moreover, an excited molecule can emit into a guided mode of the fiber through the evanescent wave [5-8].

We have developed an evanescent-wave sensor-array architecture where luminescent sensor molecules are placed at the junction of two crossed optical fibers [9-11]. The sensors are excited by laser pulses travelling in one fiber, and the resulting emission is captured by a second fiber and guided to a detector. Each sensor has a unique optical path length from the source to the detector, so the sensor luminescence arrives at the detector with a specific time delay that corresponds to the location of the sensor in the array. In addition to high spatial resolution, one major advantage of the crossed-fiber sensor architecture is the reduction of the background signal, because only the sensor luminescence is captured by the second fiber in the ideal case. We have successfully applied this sensor architecture for measuring $\mathrm{pH}$ and temperature as well as dissolved oxygen and zinc(II) concentrations in water [12-16].

Deployment of optical fiber sensors in liquid samples poses a set of issues. Sensors are fabricated by replacement of the original fiber cladding with a polymer that contains the sensor molecules. For short sensor response times, this polymer should have sufficient porosity for the efficient analyte penetration to the sensor molecule sites. However, increased porosity causes leaching of the sensor dyes if these are not covalently attached to polymer replacement cladding (which, preferably, should be attached to the fiber core). While our 
earlier experiments with the zinc-sensitive dye FluoZin-1 that was encapsulated in a polymer replacement cladding were successful [15], the sensor assembly had a limited deployment time of 3 months. Moreover, particularly in our crossed-fiber sensor platform, swelling of the cladding itself may cause changes of the luminescence signal, resulting in increased measurement errors.

Hence, covalently attachment of the sensor dyes in a highly porous polymer is necessary. However, retaining both the sensing ability for the metal ions of interest and preserving the luminescence indication of a sensing event is challenging. An additional problem for luminescent sensor dyes is their photostability. Thus, in spite of the measurement advantages afforded by the luminescent crossed-fiber sensor platform, the ability to use absorbance-based (and nonluminescent) sensor molecules would bring advantages such as a better photostability. Moreover, a much greater number of absorbance sensor dyes are either commercially available or reported in the literature, providing more opportunities for successful modification of these for covalent attachment while retaining sensing and transduction abilities.

While a number of absorption-based optical fiber sensors have been implemented [17-22], these usually measure the light transmitted through the fiber. Thus, absorbance changes are measured against a high background signal, limiting the attainable sensitivity as in any transmission experiment. The absorption-modulated luminescence (AML) approach introduced by Potyrailo and Hieftje for the detection of ammonia [23] allows the use of absorbance sensors while retaining the benefits of luminescence measurements. In their work, an absorbance sensor and a luminescent reference were embedded into the cladding along a $40 \mathrm{~mm}$ long fiber. Presence of the analyte causes a spectral shift of the absorbance of the sensor molecule, changing how much of the excitation light is absorbed. Thus, the light intensity available to excite the reference luminophore changes as well, causing in turn a change in the luminescence intensity of the reference, allowing for indirect measurement of the sensor response.

In this paper, we present an alternate sensor architecture that is based on the absorption-modulated luminescence approach, while still retaining the advantages of the crossedfiber approach. The simplest manifestation of the system is illustrated in Figure 1. The measurement system consists of two fibers that form two crossed-fiber junctions. The "sensor region" is a stretch of optical fiber between the two crossedfiber junctions. As before, in the sensor region the core of the optical fiber is exposed to the analyte, which absorbs light propagating in the fiber core via the evanescent waves. For measurement in liquid environments, only the sensor region needs to be placed in a fluid cell. The reference crossed-fiber junctions can remain in a protected environment and are not exposed to the analyte, which removes problems hitherto encountered such as chemical interference, swelling that may cause signal drift, or leaching of unattached luminophores when porous polymer claddings are chosen for short response times (see above). The sole purpose of the reference junctions is to produce signals that reflect the intensity of light in the fiber leading to and from the sensor region. The reference junctions are not limited to luminescence signal
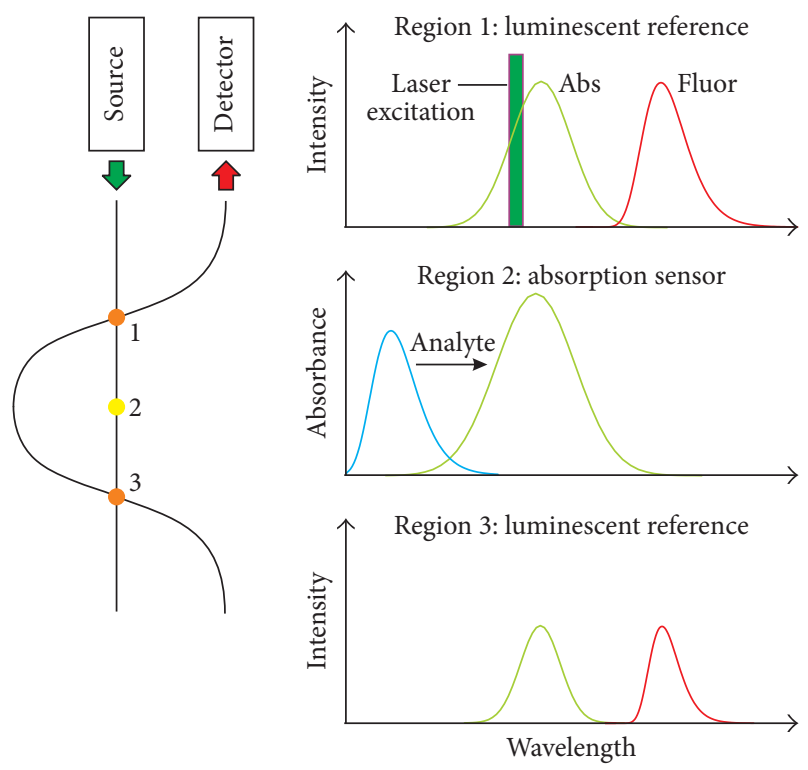

FIGURE 1: A schematic of the absorption-modulated luminescence (AML) method applied to evanescent-wave fiber optic sensors. Two reference regions are placed between a sensor region and monitor light intensity in the fiber core. The sensor region absorbs light from the fiber in the presence of analyte, reducing the luminescence of the second reference region.

generation. For example, scattering from nanoparticles may also be employed.

The measurement process is illustrated in Figure 1. The excitation wavelength is chosen such that it creates luminescence signal in the first reference region, measuring the initial light intensity in the fiber core before the sensor region. The sensor dye is chosen such that its absorption band undergoes a spectral shift in the presence of the analyte. In the scenario depicted in the Figure 1, the excitation wavelength does not overlap with the absorption band of the sensor dye when no analyte is present. The presence of the analyte shifts the sensor absorption band towards the excitation wavelength, causing increased absorption and, consequently, a reduction in the excitation light that reaches the second reference region. The second reference thus measures light intensity transmitted through the sensor region. The reverse scenario can also be employed in which the absorption band moves away from the excitation wavelength in the presence of the analyte, leading to an increase of the light intensity transmitted through the sensor region.

\section{Materials and Methods}

2.1. Reagents. Ethanol, acetone, nitric acid, 1-(2-pyridylazo)2-naphthol (PAN), hexadecyltrimethylammonium bromide (CTAB, surfactant), poly(ethylene glycol) diacrylate (PEGDA, polymer) with a number-average molecular weight of 575 , 2,2-dimethoxy-2-phenylacetophenone (DMPA, photoinitiator), and a zinc standard for atomic absorption spectroscopy were purchased from Sigma-Aldrich (Milwaukee, Wisconsin, USA). MOPS acid and MOPS sodium salt were purchased 

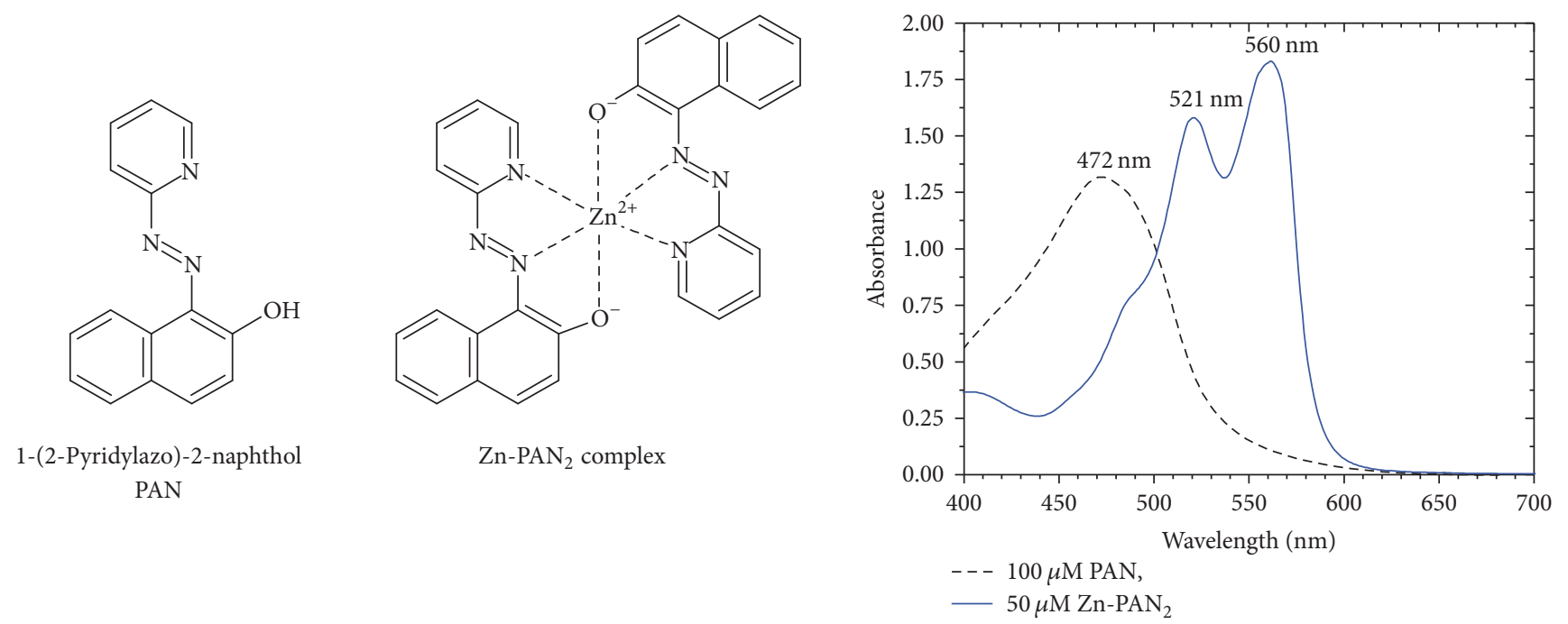

Figure 2: Chemical structures and absorption spectra of PAN and zinc-PAN ${ }_{2}$ complex.

from Alfa Aesar (Ward Hill, Massachusetts, USA). Lasergrade rhodamine 110 was purchased from Exciton (Dayton, Ohio, USA). A 1\% (w/v) aqueous suspension of fluorescent polystyrene microspheres, having a $1.01 \mu \mathrm{m}$ mean diameter and loaded with "Envy Green" fluorophore, was purchased from Bangs Laboratories (Fishers, Indiana, USA). Also, ultrapure water was used throughout this work.

2.2. Choice of Sensor and Reference Dyes. Pyridylazo-naphthol (PAN) is a well-known azo dye that forms colored complexes with metal ions, including copper, cobalt, cadmium, nickel, and zinc [24-27]. The structures and absorbance spectra of PAN and the $\mathrm{Zn}-\mathrm{PAN}_{2}$ complex are given in Figure 2. The apo-form of PAN has an orange color below pH 8 with a strong absorption near $470 \mathrm{~nm}$ [27]. PAN forms a deep red-magenta colored complex with zinc having two absorption peaks near 520 and $560 \mathrm{~nm}$. PAN was used here as an absorbance sensor for zinc(II) because it exhibits a large spectral change at $560 \mathrm{~nm}$ (Figure 2). Microspheres doped with a fluorophore with an excitation bands overlapping with the $560 \mathrm{~nm}$ excitation wavelength were used to construct the reference regions.

2.3. Sample Preparation. A stock solution of $4 \mathrm{mM}$ PAN was prepared in denatured absolute ethanol, and a stock buffer solution containing $8 \mathrm{mM} \mathrm{CTAB}$ and $5 \mathrm{mM}$ MOPS buffer at $\mathrm{pH} 7$ was prepared. The sample solutions were prepared in the following sequence [27] in Class A volumetric flasks. First, a portion of the buffer solution was added to the flask. Next, the zinc standard was added so the initial zinc concentration ranged from $0 \mu \mathrm{M}$ to $50 \mu \mathrm{M}$ in the sample. Then, a portion of the PAN stock solution was added so the initial PAN concentration was $100 \mu \mathrm{M}$ in the sample. The volumetric flask was diluted to the mark with stock buffer solution and inverted twenty times. The solutions were allowed to sit for 30 minutes to allow formation of the zinc-PAN ${ }_{2}$ complex. The samples were injected into the fluid cell using a disposable syringe.
2.4. Fluid Cell. The fluid cell in Figure 3 was fabricated from borosilicate glass tubing with a $4 \mathrm{~mm}$ outer diameter and $2 \mathrm{~mm}$ inner diameter. The central tube was cut to a length of $12 \mathrm{~cm}$, and the inlet and outlet ports were installed on the central tube using standard glassblowing techniques. The fluid cell was washed with Citranox detergent to remove any foreign matter. Inert fluorinated ethylene propylene (FEP) tubing with a Luer adapter from Hamilton (Reno, Nevada, USA) was installed onto the ports to allow injection of solutions with a disposable syringe.

2.5. Fiber Preparation. Bare step-index multimode optical fiber with high-OH content, $400 \mu \mathrm{m}$ core diameter, and 0.37 NA was purchased from Thor Labs (Newton, New Jersey, USA). The illumination fiber was constructed from a $15.5 \mathrm{~m}$ length of fiber. One end of the fiber was terminated with an SMA connector for coupling with the light source. The fiber has an overfilled launch condition at the laser, so a $10 \mathrm{~m}$ section was reserved to deplete light in high-order "lossy" modes. The distal end of the fiber was cleaved and submerged in a glycerol solution, which has a refractive index that exceeds the fiber core, to suppress the backreflection of the laser pulse. The detection fibers were constructed from $5 \mathrm{~m}$ and $10 \mathrm{~m}$ lengths of fiber and terminated with an SMA connector for coupling to the detectors.

The illumination fiber had three stripped sections. The sensor region itself was $8 \mathrm{~cm}$ long and located between two smaller sections of stripped fiber (R1 and R2 in Figure 3). The reference regions were $5 \mathrm{~mm}$ long and separated from the sensor region by a distance of $12 \mathrm{~cm}$. Also, each detection fiber had a single stripped section for creating the reference region that was $5 \mathrm{~mm}$ long and located $2 \mathrm{~cm}$ from the distal end.

The buffer and cladding layers of the optical fiber were thermally removed to expose sections of the fiber core using a metal mask. The mask was fabricated from $0.5 \mathrm{~mm}$ thick piece of aluminum with an $8.5 \mathrm{~cm}$ long, $0.5 \mathrm{~cm}$ wide slot for the sensor region, and a $7 \mathrm{~mm}$ diameter hole for the reference 


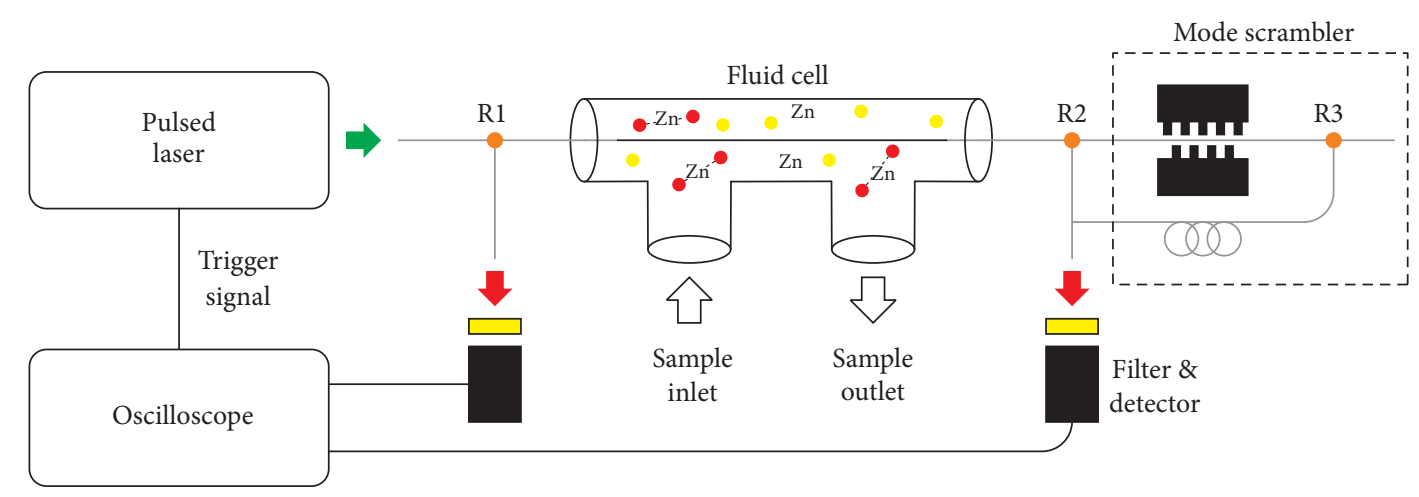

FIGURE 3: Illustration of optical setup for AML measurements. The dashed box includes components for a separate experiment involving a mode scrambler.

regions. The fiber was positioned on top of the mask, centered over the slot, and secured with transparent tape. The buffer and cladding layers were thermally removed using a butane lighter held a few centimeters below the mask. The fiber was not exposed to the flame for longer than ten seconds to prevent ignition of the coating or deformation of the fiber core. The fiber was carefully removed from the mask, and the stripped sections of fiber were gently cleaned with an acetonesoaked laboratory tissue.

The sensor region was carefully inserted into the fluid cell. The fiber was fixed and sealed to the fluid cell using Norland Optical Adhesive \#61 (Cranbury, New Jersey, USA). A small amount of the adhesive was injected into one end of the fluid cell with a syringe and blunt-tipped needle and cured for five minutes using $365 \mathrm{~nm}$ light from a Spectroline handheld UV lamp (Westbury, New York, USA). Next, a slight tension was applied to the fiber to ensure that the stripped section remained taut as the other end of the cell was sealed in the same manner. The optical adhesive was aged for 60 hours at room temperature. The fluid cell and sensor fiber were cleaned overnight with $2 \%(\mathrm{v} / \mathrm{v})$ nitric acid and rinsed with a copious amount of water.

2.6. Reference Junctions. A crossed-fiber junction was created with a polymer coating containing the fluorescent microsphere for each reference region. A polypropylene block was used for mounting the fibers and was machined with two sets of shallow grooves at a 90-degree angle forming an equidistant cross. First, the stripped section of the illumination fiber was mounted into the block and temporarily secured with transparent tape. Next, the stripped section of the detection fiber was placed on top of the illumination fiber at a 90-degree angle, positioned into the second set of grooves in the block, and temporarily secured with transparent tape. A cover was installed on the block, fixing the position of both fibers, and the tape was removed.

A precursor solution was prepared in a vial. First, the MEHQ inhibitor was removed from a portion of PEGDA using a SDHR-4 column from Scientific Polymer Products (Ontario, New York, USA). Next, 3 mg DMPA (photoinitiator, $1 \% \mathrm{w} / \mathrm{v}$ ) was added to $300 \mu \mathrm{L}$ of PEGDA and thoroughly mixed. Then, $100 \mu \mathrm{L}$ of water $(20 \% \mathrm{v} / \mathrm{v})$ and $100 \mu \mathrm{L}$ of the "Envy Green" microsphere suspension (20\% v/v, received as $1 \%$ solids) were added to the vial and thoroughly mixed. The precursor solution was sonicated for 3 minutes prior to application. A $2 \mu \mathrm{L}$ drop of the precursor solution was applied to the crossed-fiber junction with a micropipette, and the polymer was cured for five minutes using $365 \mathrm{~nm}$ light from the Spectroline handheld lamp.

2.7. Mode Scrambling. A separate experiment involved a third reference region, $\mathrm{R} 3$, to examine if the sensor region depleted higher-order modes from the illumination fiber. The additional components are shown in the box with a dashed outline in Figure 3. The third reference region was fabricated in the same manner as described in the previous section, except that the detection fiber was $10 \mathrm{~m}$ longer than the detection fiber for R2. The additional fiber length created an optical delay to temporarily separate the luminescence signals recorded by the oscilloscope. A Newport FM-1 mode scrambler (Irvine, California, USA) was used to precisely control the distorting force applied to the fiber, which causes mode mixing in the fiber core, and was placed between R2 and R3. The initial position of the mode scrambler was adjusted such that the illumination fiber was taut in the mode scrambler slot.

First, the fluid cell was filled with a solution containing $50 \mu \mathrm{M}$ of the $\mathrm{Zn}-\mathrm{PAN}_{2}$ complex, and waveforms were recorded as increasing force was applied to the fiber. Next, the illumination fiber was cut back and R3 was replaced in case the mode scrambler created a permanent deformation of the fiber. The fluid cell was then filled with ultrapure water, and the same experiment was repeated.

2.8. Light Source. The light source consisted of Photon Technology International GL3200 and GL302 nitrogen-pumped dye laser (Edison, New Jersey, USA) emitting $555 \mathrm{~nm}$ pulses with $10 \mathrm{mM}$ rhodamine 110 in ethanol. This wavelength has strong overlap with the absorption of both the $\mathrm{Zn}-\mathrm{PAN}_{2}$ complex and the fluorescent microspheres in the reference regions. The dye laser contains a photodiode that generated a trigger signal for the oscilloscope. 
2.9. Detector. The luminescence signals from the reference regions were measured with Hamamatsu H10721 PMT detectors (Hamamatsu City, Shizuoka, Japan) equipped with an SMA collimator lens and interference filter with a $600 \mathrm{~nm}$ central wavelength and $50 \mathrm{~nm}$ spectral bandpass (Edmund Optics, Barrington, Illinois, USA). The waveforms were recorded and internally averaged using a LeCroy $1 \mathrm{GHz}$ digitizing oscilloscope (Chestnut Ridge, New York, USA) and the laser photodiode trigger signal. The oscilloscope averaged the waveforms from 250 laser pulses. The detection fibers were measured with individual detectors so that each channel could be scaled to maximize the resolution of the analogto-digital converter (ADC) in the oscilloscope. The averaged waveforms were numerically integrated, and the attenuation values were calculated using a LabVIEW routine (National Instruments, Austin, Texas, USA).

\section{Results and Discussion}

Samples containing $100 \mu \mathrm{M}$ PAN and different amounts of zinc were placed in the fluid cell, and the luminescence signals from the reference region were recorded. Three replicate measurements were performed for each sample. Example waveforms for the $0 \mu \mathrm{M}$ and $50 \mu \mathrm{M}$ zinc samples are shown in Figure 4(a). The intensity of R1, which is proximal to the absorption sensor, varies from fluctuations in the pulsed light source. The intensities of R2 and R3 decreased, resulting from evanescent absorption of light in the illumination fiber by the $\mathrm{Zn}-\mathrm{PAN}_{2}$ complex. The intensity of R3 was not used in the analysis as no mode scrambling was employed in this experiment.

The waveform peaks were numerically integrated, and the integrated values, denoted as $I_{\mathrm{R} 1}$ and $I_{\mathrm{R} 2}$, were used to calculate the optical attenuation caused from the sample.

$$
\text { Attenuation }=-\log \left(\frac{I_{\mathrm{R} 2}}{I_{\mathrm{R} 1}}\right) .
$$

Plots of the attenuation versus zinc(II) concentration are shown in Figures 4(b) and 4(c). The response is nonlinear over the investigated zinc(II) concentration range of $0 \mu \mathrm{M}$ to $50 \mu \mathrm{M}$. This data was truncated, and a linear response was observed up to $20 \mu \mathrm{M}$ zinc(II).

The nonlinear response of the absorption sensor is not the result of common deviations from the Beer-Lambert Law. The dye laser has a narrow spectral output with a linewidth specification of $0.04 \mathrm{~nm}$, so errors caused by polychromatic light can be excluded. Instrumental deviations caused by the presence stray light and low sample transmission can be neglected because the optical intensities $\left(I\right.$ and $I_{0}$ ) are not measured directly through the sample. Instead, the intensity in the illumination fiber was indirectly measured via the luminescence of the reference regions over a 50 ns time interval where background light levels are low. Also, the luminescence signals of R2 (and R3) were relatively strong, and the high absorbance (near 2.0) of the $\mathrm{Zn}-\mathrm{PAN}_{2}$ complex did not extinguish excitation of the reference regions.

Mode depletion is one possible explanation for the nonlinear response. The effect has been observed in work with evanescent fiber sensors that performs a transmission measurement with a single optical fiber $[28,29]$. Formally, a mode is a single solution of Maxwell's equation for a given system with a set of boundary conditions [30]. A mode can be imagined as a path in which light travels down the fiber core by total internal reflection. Axial modes consist of light travelling directly through the fiber core along the fiber axis. Low-order modes consist of light propagating down the fiber with an incident angle near the fiber axis and undergo a fewer total internal reflections per unit length, and high-order modes consist of light propagating down the fiber with an incident angle near the critical angle and undergo many total internal reflections per unit length. The fiber used in this work has an approximate $\mathrm{V}$-number of 939 at $555 \mathrm{~nm}$ and supports approximately 440,000 guided modes.

The evanescent wave can be used to couple light in and out of the fiber core, as was utilized here for both the sensor and reference regions. The penetration depth of each mode depends on the wavelength of light, the refractive indices of the core and cladding at the specified wavelength, and the angle of incidence [31]. High-order modes have a deeper penetration depth, and therefore high-order modes will have a greater probability of evanescent absorption by a given sample at the exposed fiber core boundary than low-order modes. Additionally, the strong absorptivity of the $\mathrm{Zn}-\mathrm{PAN}_{2}$ complex may have depleted light in the higher-order modes in the illumination fiber that can be interrogated by the reference regions, as illustrated in Figure 5. The topic of mode depletion was examined in an experiment with the third reference region and a mode scrambler.

The mode scrambler used in this work creates precise distortions in the fiber geometry with two corrugated plates, and the distance between the two plates can be adjusted with an incrementing dial. Consequently, the microbends induced on the illumination fiber cause mode coupling and redistribution of light in the fiber core. The spacing between the two corrugated plates was reduced by $10 \mu \mathrm{m}$ for each increment of the adjustment dial.

If strong absorption by the sensor depleted light in highorder modes that are interrogated by the reference region, then scrambling is expected to restore light in these modes, as observable via a reference region. As shown in Figure 3 a mode scrambler was placed after the second reference region $\mathrm{R} 2$, and a third reference junction R3 was used to monitor the effect of mode scrambling. The luminescence signals of R2 and R3 were recorded at different increments of the mode scrambler while the fluid cell was filled with either water or $50 \mu \mathrm{m} \mathrm{Zn-PAN}{ }_{2}$ solution. The ratios of the integrated intensities of R3 and R2, respectively, were computed and plotted against the increment of the mode scrambler, as shown in Figure 6.

The intensity ratio increases for the first ten increments of the mode scrambler when the cell is filled with dye solution. This indicates that some of the high-order modes that can be interrogated by the reference regions were depleted by the strong absorbance of the complex in the cell, and these modes could be replenished by the mode scrambler. Then, the intensity ratio is relatively constant for increments 10 through 20 where mode scrambling nets no improvement. Finally, the 

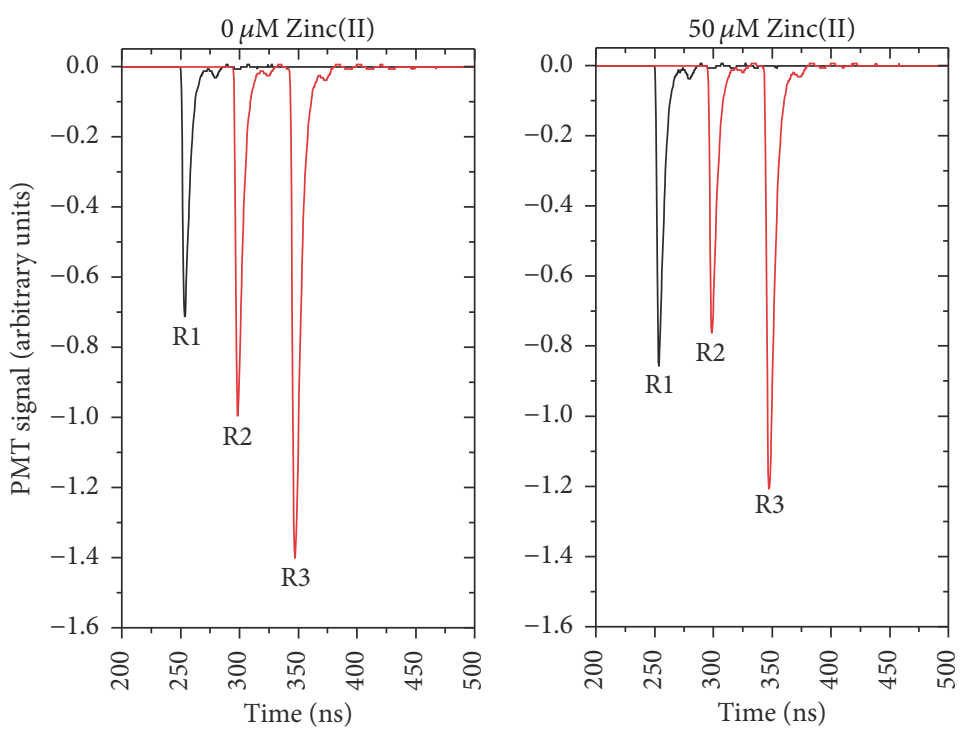

(a)

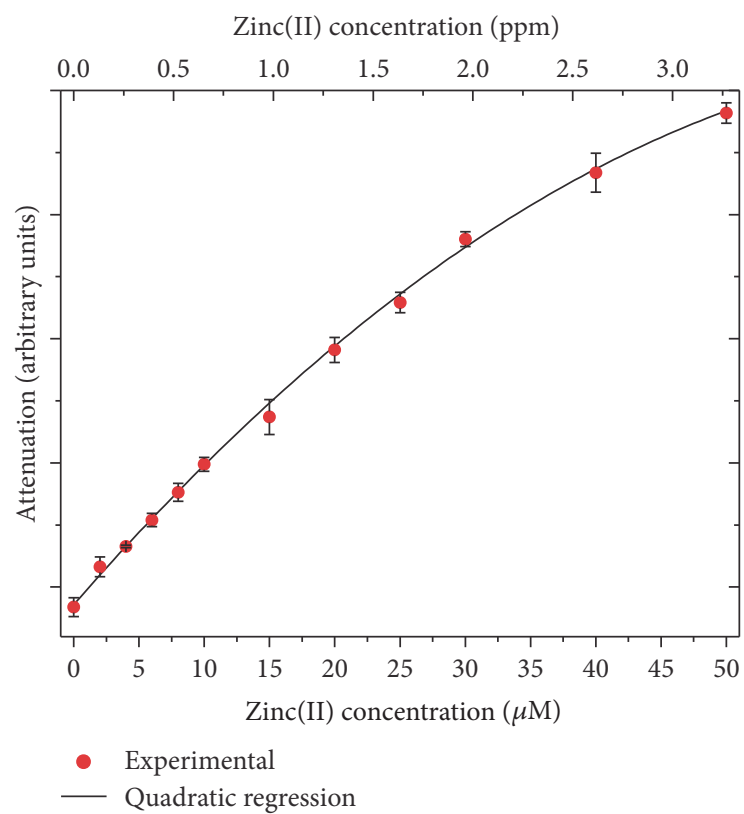

(b)

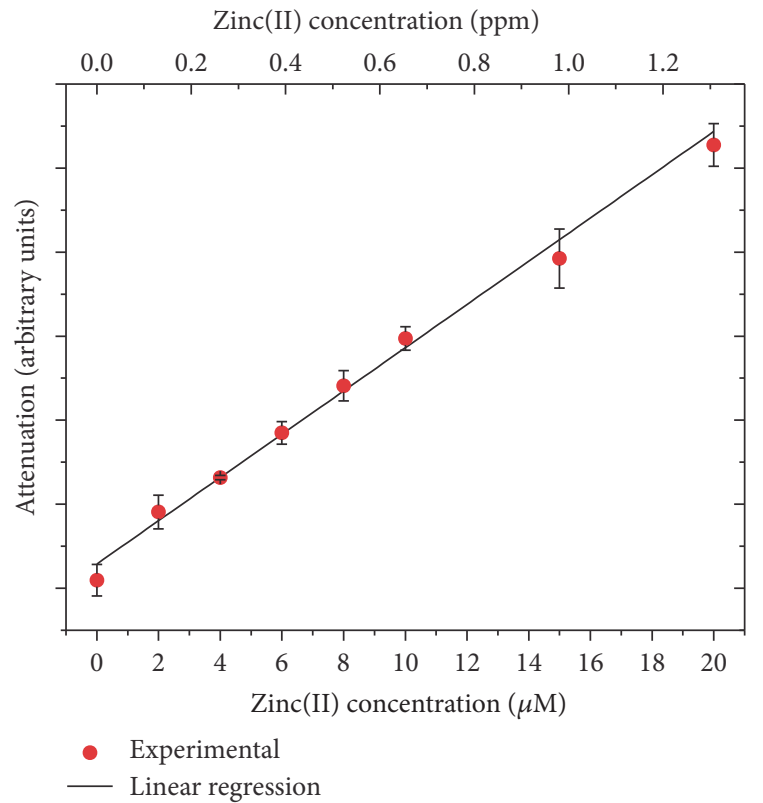

(c)

FIGURE 4: Attenuation measurement with $100 \mu \mathrm{M}$ PAN. (a) Example waveforms from measurements with no zinc and with $50 \mu \mathrm{M}$ zinc. (b) Plot of the attenuation versus zinc concentration with quadratic regression. (c) Plot of truncated data with linear regression. The errors bars represent the $95 \%$ confidence interval with three replicate measurements. Also, the title graph on the right in Figure 4 a should be changed to $50 \mu \mathrm{M}$ for consistency.

intensity ratio decreases sharply above increment 20 where light was visibly radiating from the fiber at the mode scrambler, and guided modes were converted to lossy modes.

The intensity ratio is relatively constant for the first eighteen increments when the cell is filled with water. This suggests that no net improvement is observed when the sample does not appreciably absorb light in the illumination fiber. The intensity ratio increases slightly above increment 25 , which suggests that the relatively large clamping force is distributing light into lossy modes. The intensity ratio decreases above increment 40 , but at a slower rate than the zinc complex. Also, light was visibly radiating from the fiber above increment 41. Finally, the clamping force fractured the fiber core at increment 55.

The difference between the two curves suggests that strong absorption in the sensor region depletes a noticeable fraction of light in the guided modes of the illumination fiber. Moreover, it is the high-order modes that cause the largest 

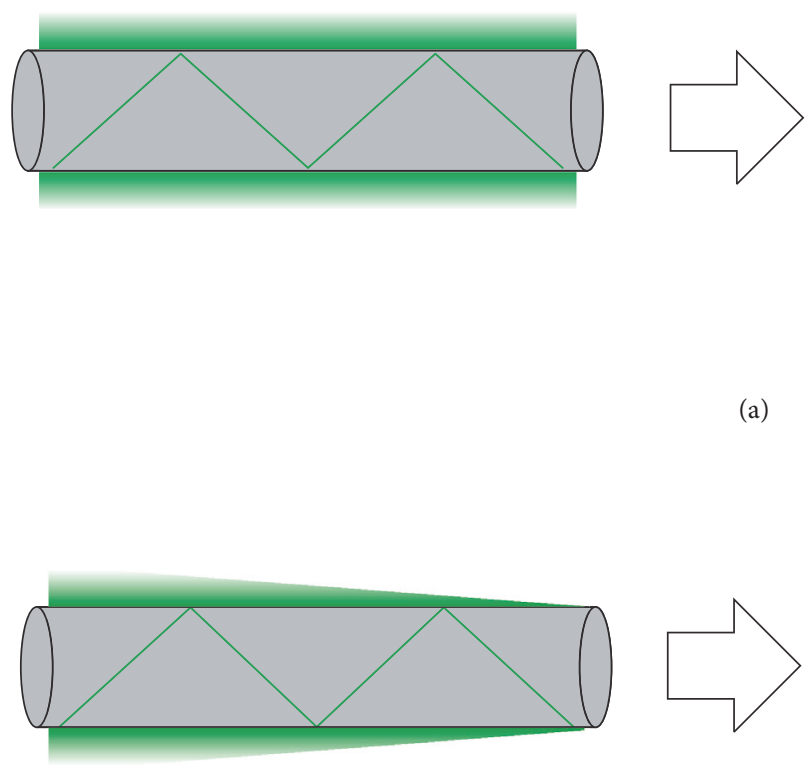

(b)

(a)
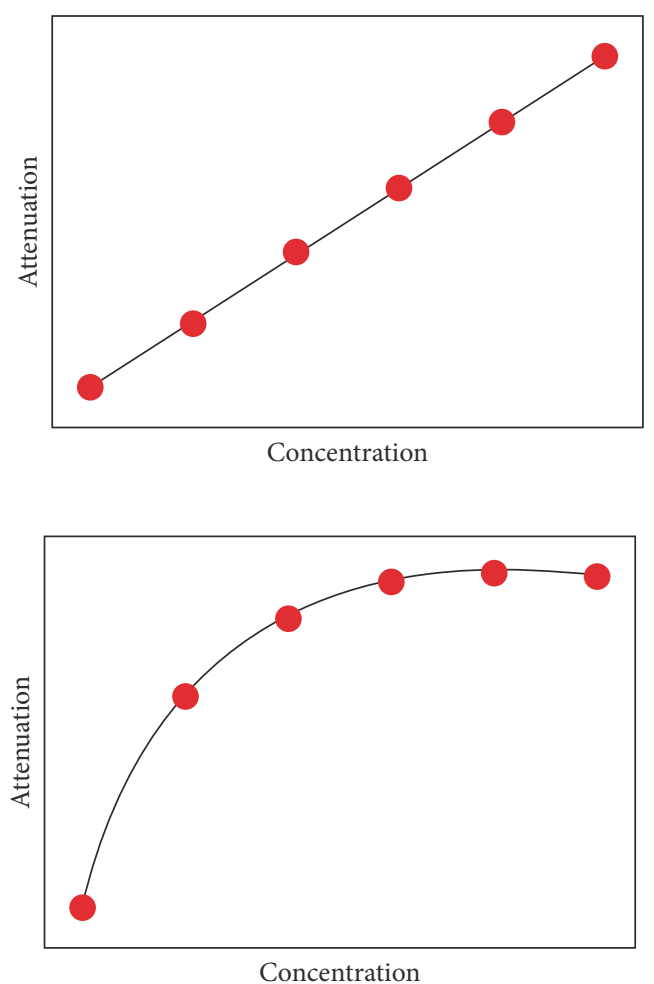

FiguRE 5: Illustration of the evanescent wave generated at the boundary of the fiber core and the effect of mode depletion of high-order modes by an absorbing sample. (a) Linear response obtained when light is equally absorbed by all modes. (b) Nonlinear response obtained when high-order modes are depleted.

FM-1 clamping distance $(\mu \mathrm{m})$

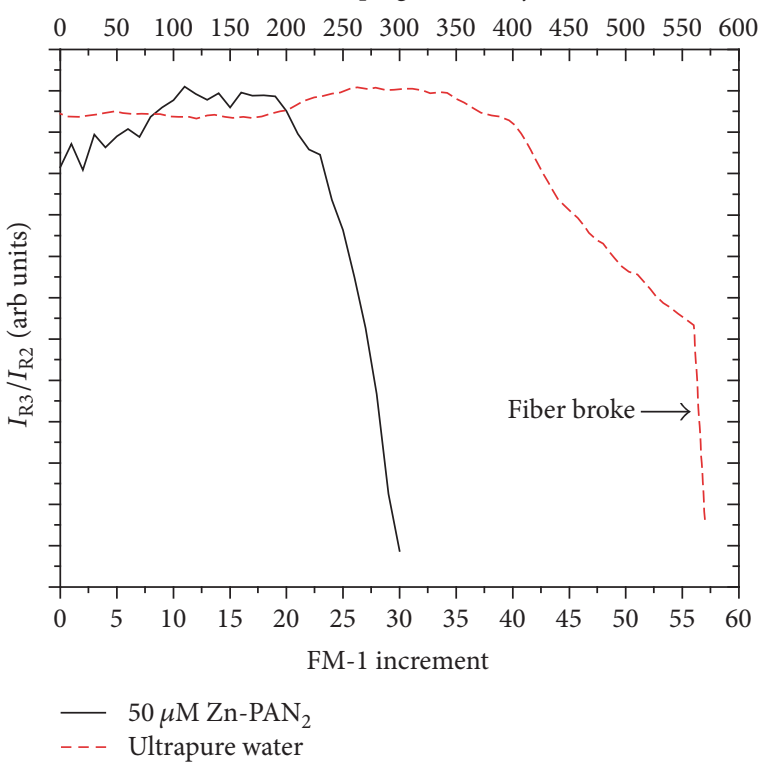

FIGURE 6: A plot of the intensity ratio of reference regions R3 and R2 versus the as-increased pressure is applied to the illumination fiber by the mode scrambler. The $\mathrm{Zn}-\mathrm{PAN}_{2}$ complex absorbs a significant fraction of light in the illumination fiber that can be probed by the reference regions. contribution to the luminescence signals in the reference junctions. In effect, the depletion of predominately highorder modes is amplified in the reference junction R2, because their absence causes a reduction in reference signal generation.

The mode scrambler was able to repopulate some highorder modes (at the expense of low-order modes), which results in the increase in light intensity recorded by R3. Additional pressure on the fiber by the mode scrambler, however, led to a steep drop in the R3 intensity where light was visibly radiating from the illumination fiber. Interestingly, when the fluid cell was filled with water only, the R3 intensity remains largely unchanged for mode scrambler increments that already caused a sharp drop in the R3 signal when the Zn$\mathrm{PAN}_{2}$ solution was present. One reason could be that for the latter case the mode scrambler depletes low-order modes to an extent that little intensity is left (the low-order modes constitute an "optical intensity reservoir") to repopulate the high-order modes. On the other hand, in the case of pure water in the fluid cell, no mode depletion occurs in the sensor region occurs, meaning that the mode scrambler just redistributes intensity among modes until the fiber is deformed to such an extent that large losses occur in the mode scrambler.

The sensitivity of the AML technique was compared with a conventional absorption measurement using a $1 \mathrm{~cm}$ cuvette, 


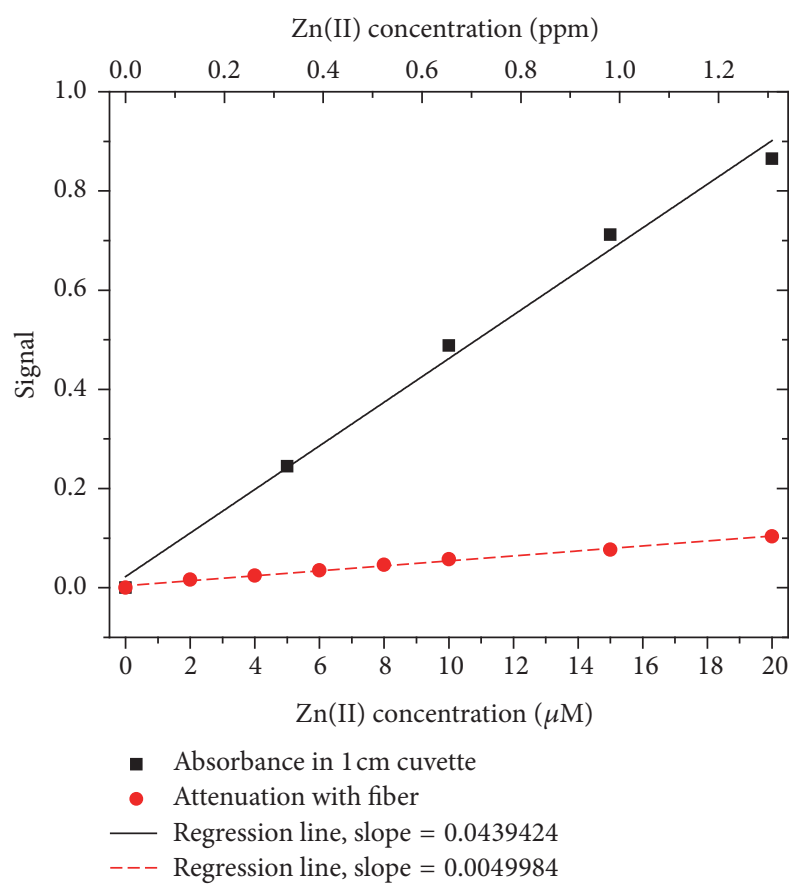

Figure 7: A plot of the signal attenuation at $555 \mathrm{~nm}$ resulting from the complex formed with varying zinc(II) concentrations for an AML measurement and a conventional absorbance measurement. The sensitivity of the conventional measurement is 8.69 times larger than the AML measurement based on the slopes of the regression lines.

and the result is shown in Figure 7. As expected, the sensitivity of the optical fiber measurement is less than that of the $1 \mathrm{~cm}$ cuvette measurement by a factor of approximately one-tenth. However, this value should be placed in context of the measurement volume. A rough estimate using as the evanescentfield-penetration depth the value of $1 \mu \mathrm{m}$ ( 2 times the wavelength of the probe light used), the fiber radius of $200 \mu \mathrm{m}$, and the length of the absorption section of $8 \mathrm{~cm}$ (with sensing occurring on the full circumference of the fiber in that section) yields a probe volume of $3.2 \pi \times 10^{-5} \mathrm{~cm}^{3}$. For the $1 \mathrm{~cm}$ cuvette and a circular beam with a radius of $1 \mathrm{~mm}$, the probe volume is $\pi \times 10^{-2} \mathrm{~cm}^{3}$, meaning that the sensing volume for the optical fiber measurement is smaller by a factor of $3.2 \times$ $10^{-3}$. This demonstrates that overall the sensing capability of the method is quite satisfactory.

\section{Summary and Conclusions}

We successfully implemented a technique, referred to as absorption-modulated luminescence that allows for use of nonluminescent sensor molecules in the crossed-fiber, evanescent-wave sensor architecture. Presence of the analyte changes the absorbance of sensor dye and changes the transmission of light through the optical fiber. The transmission changes are measured via the ratio of the luminescence signal from two luminescent crossed-fiber sensor junctions, one located before and one after the stretch of optical fiber where evanescent absorption occurs.
The method and apparatus were tested with the zincsensitive dye PAN, and linear response was measured for zinc(II) concentration up to $20 \mu \mathrm{M}$. For higher concentration the response becomes nonlinear, in agreement with the results from a single-fiber evanescent-absorption measurement reported earlier [29]. The nonlinearity was attributed to high-order mode depletion, which was verified by the observed repopulation of high-order modes with a mode scrambler that was positioned after the second crossed-fiber junction.

The AML-Crossed-Fiber technique retains the advantage of low background of the signals generated at the luminescent crossed-fiber junctions used for the measurement, while allowing for the use of nonluminescent sensor dyes, as long as these responds to sensing events by a spectral shift of a characteristic absorption band. Moreover, only the sensor dyes have to be in contact with the sample, whereas the crossedfiber measurement junctions can be placed in separate, benign environments.

This separation of the sensing region from the signalgeneration regions makes the method quite flexible and adaptable. Firstly, the signal generation in the crossed-fiber junctions is not limited to luminescent dyes. Quantum dots, which typically have broad excitation spectra, narrow emission spectra, high quantum yield, and high photostability, may be employed. Scattering centers may also be used for reference signal generation. Secondly, for absorbance sensor dyes with lower molar extinction coefficients, the length of the absorbance sensor section may be increased to retain or improve sensitivities. Only a single excitation wavelength is needed, which can be tuned to the chosen sensor absorption features. Thirdly, multiple fibers can be routed through the fluid cell, with each fiber carrying different absorbance sensors for various target analytes. Moreover, if the sensor dyes are also luminescent, in addition to the absorbance change, the luminescence signal would be coupled back into the fiber which could be measured at either of or both of the crossedfiber junctions where detectors are placed at the other ends of the crossing fibers with the appropriate spectral filters.

\section{Conflicts of Interest}

The authors declare that they have no conflicts of interest.

\section{Acknowledgments}

The authors gratefully acknowledge funding from the University of Wisconsin-Milwaukee Research Growth Initiative. Additional funding came from a Translational Grant by the Southeast Wisconsin Applied Chemistry Center of Excellence at the University of Wisconsin-Milwaukee. The authors thank Dr. Robert Olsson for advice and loan of instrument components and Mr. Neal Korfhage for the fabrication of the fluid cell.

\section{References}

[1] J. B. Faria, "A theoretical analysis of the bifurcated fiber bundle displacement sensor," IEEE Transactions on Instrumentation and Measurement, vol. 47, no. 3, pp. 742-747, 1998. 
[2] D. J. Sterling, Technician's Guide to Fiber Optics, vol. 384, Delmar Cengage Learning, 4th edition, 2003.

[3] A. K. Ghosh and P. K. Paul, "Alignment considerations in extrinsic fiber-optic sensors," Applied Optics, vol. 36, no. 25, pp. 6256$6263,1997$.

[4] X.-D. Wang and O. S. Wolfbeis, "Fiber-optic chemical sensors and biosensors (2013-2015)," Analytical Chemistry, vol. 88, no. 1, pp. 203-227, 2016.

[5] D. Marcuse, "Launching light into fiber cores from sources located in the cladding," Journal of Lightwave Technology, vol. 6, no. 8, pp. 1273-1279, 1988.

[6] C. K. Carniglia, L. Mandel, and K. H. Drexhage, "Absorption and emission of evanescent photons," Journal of the Optical Society of America, vol. 62, no. 4, article 479, 1972.

[7] D. A. Christensen, J. D. Andrade, J. Wang et al., "Evanescentwave coupling of fluorescence into guided modes: FDTD analysis," in Chemical, Biochemical, and Environmental Fiber Sensors, SPIE-The International Society for Optical Engineering, 1990.

[8] J. Ma and W. J. Bock, "Dramatic performance enhancement of evanescent-wave multimode fiber fluorometer using nonLambertian light diffuser," Optics Express, vol. 15, no. 25, pp. 16457-16470, 2007.

[9] B. J. Prince, A. W. Schwabacher, and P. Geissinger, "A readout scheme providing high spatial resolution for distributed fluorescent sensors on optical fibers," Analytical Chemistry, vol. 73, no. 5, pp. 1007-1015, 2001.

[10] B. J. Prince, N. T. Kaltcheva, A. W. Schwabacher, and P. Geissinger, "Fluorescent fiber-optic sensor arrays probed utilizing evanescent fiber-fiber coupling," Applied Spectroscopy, vol. 55, no. 8, pp. 1018-1024, 2001.

[11] P. E. Henning, A. Benko, A. W. Schwabacher, P. Geissinger, and R. J. Olsson, "Apparatus and methods for optical time-offlight discrimination in combinatorial library analysis," Review of Scientific Instruments, vol. 76, no. 6, pp. 622201-622208, 2005.

[12] P. Geissinger and M. V. Rigo, "Measurement and optimization of metal-nanoparticle-induced luminescence enhancement factors in a crossed-optical fiber configuration," Journal of Nanomaterials, vol. 2010, Article ID 396214, 11 pages, 2010.

[13] M. V. Rigo, R. J. Olsson, and P. Geissinger, "Crossed-opticalfiber oxygen sensors with intensity and temperature referencing for use in high-spatial-resolution sensor arrays," Sensors and Transducers Journal, vol. 113, no. 2, pp. 18-32, 2010.

[14] P. E. Henning, M. V. Rigo, and P. Geissinger, "Fabrication of a porous fiber cladding material using microsphere templating for improved response time with fiber optic sensor arrays," The Scientific World Journal, vol. 2012, Article ID 876106, 7 pages, 2012.

[15] S. Kopitzke and P. Geissinger, "An optical fiber-based sensor array for the monitoring of zinc and copper ions in aqueous environments," Sensors, vol. 14, no. 2, pp. 3077-3094, 2014.

[16] M. V. Rigo and P. Geissinger, "Crossed optical fiber sensor arrays for high-spatial-resolution sensing: application to dissolved oxygen concentration measurements," Journal of Sensors, vol. 2012, Article ID 464092, 10 pages, 2012.

[17] P. H. Paul and G. Kychakoff, "Fiber-optic evanescent field absorption sensor," Applied Physics Letters, vol. 51, no. 1, pp. 1214, 1987.

[18] J. Bürck, J.-P. Conzen, and H.-J. Ache, "A fiber optic evanescent field absorption sensor for monitoring organic contaminants in water," Fresenius' Journal of Analytical Chemistry, vol. 342, no. 4-5, pp. 394-400, 1992.
[19] R. A. Potyrailo, S. E. Hobbs, and G. M. Hieftje, "Near-ultraviolet evanescent-wave absorption sensor based on a multimode optical fiber," Analytical Chemistry, vol. 70, no. 8, pp. 1639-1645, 1998.

[20] B. D. Gupta and S. K. Khijwania, "Experimental studies on the response of the fiber optic evanescent field absorption sensor," Fiber and Integrated Optics, vol. 17, no. 1, pp. 63-73, 1998.

[21] S. K. Khijwania and B. D. Gupta, "Fiber optic evanescent field absorption sensor: effect of fiber parameters and geometry of the probe," Optical and Quantum Electronics, vol. 31, no. 8, pp. 625-636, 1999.

[22] R. A. Potyrailo, V. P. Ruddy, and G. M. Hieftje, "Use of analytemodulated modal power distribution in multimode optical fibers for simultaneous single-wavelength evanescent-wave refractometry and spectrometry," Analytical Chemistry, vol. 71, no. 21, pp. 4956-4964, 1999.

[23] R. A. Potyrailo and G. M. Hieftje, "Optical time-of-flight chemical detection: absorption-modulated fluorescence for spatially resolved analyte mapping in a bidirectional distributed fiberoptic sensor," Analytical Chemistry, vol. 70, no. 16, pp. 34073412, 1998.

[24] K. L. Cheng and R. H. Bray, "1-(2-pyridylazo)-2-naphthol as a possible analytical reagent," Analytical Chemistry, vol. 27, no. 5, pp. 782-785, 1955.

[25] A. Corsini, I. M.-L. Yih, Q. Fernando, and H. Freiser, "Potentiometric investigation of the metal complexes of 1-(2-pyridylazo)2-naphthol and 4-(2-pyridylazo)-resorcinol," Analytical Chemistry, vol. 34, no. 9, pp. 1090-1093, 1962.

[26] P. Hooft, H. Van De Voorde, and P. Van Dijck, "A more sensitive modification of the zinc test for seminal traces suitable for stable test paper strips," Forensic Science International, vol. 53, no. 2, pp. 131-133, 1992.

[27] J. Krystek, J. Kobylecka, and B. Ptaszynski, "Spectrophotometric determination of zinc with 1-(2-Pyridylazo)-2-naphthol and cetyltrimethylammonium bromide in insulin," Chemia Analityczna, vol. 38, no. 5, pp. 607-612, 1993.

[28] F. P. Payne and Z. M. Hale, "Deviation from beer's law in multimode optical fibre evanescent field sensors," International Journal of Optoelectronics, vol. 8, no. 5-6, pp. 743-748, 1993.

[29] M. D. DeGrandpre and L. W. Burgess, "Long path fiber-optic sensor for evanescent field absorbance measurements," Analytical Chemistry, vol. 60, no. 23, pp. 2582-2586, 1988.

[30] F. de Fornel, Evanescent Waves: From Newtonian Optics to Atomic Optics, vol. 73 of Springer Series in Optical Sciences, Springer, 2001.

[31] N. J. Harrick, Internal Reflection Spectroscopy, Intersciences Publishers, London, UK, 1967. 


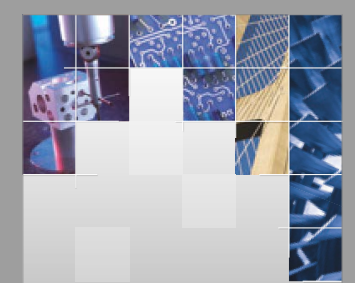

\section{Enfincering}
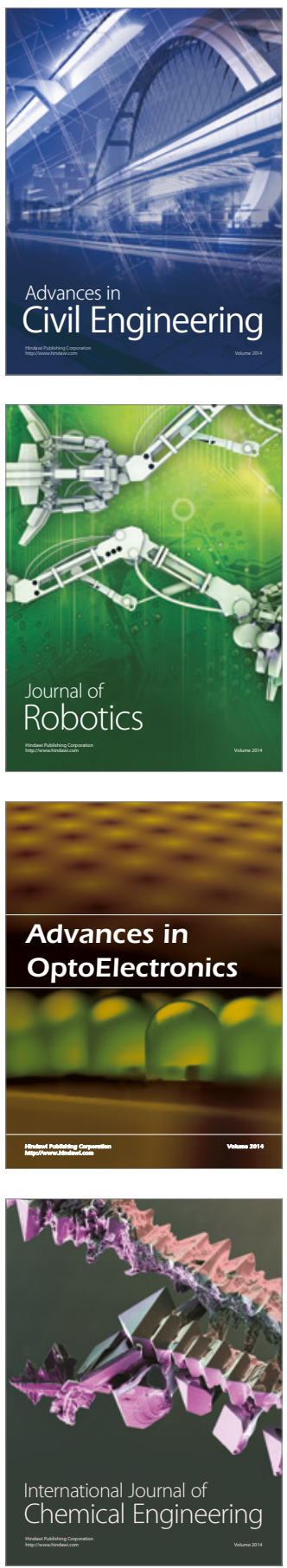

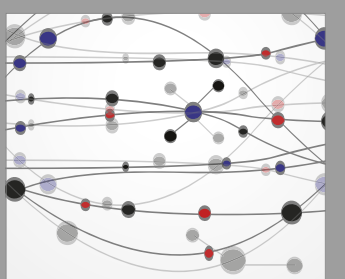

The Scientific World Journal

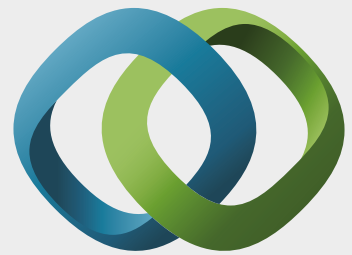

\section{Hindawi}

Submit your manuscripts at

https://www.hindawi.com
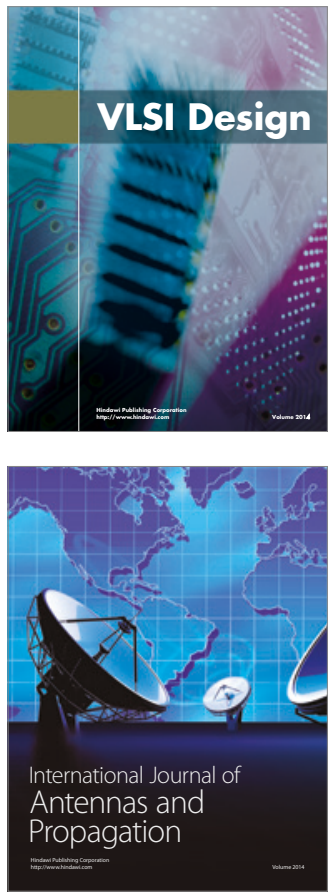

\section{Rotating}

Machinery
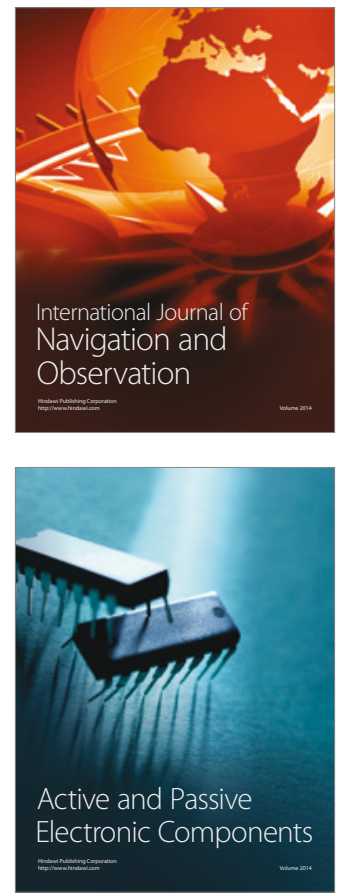
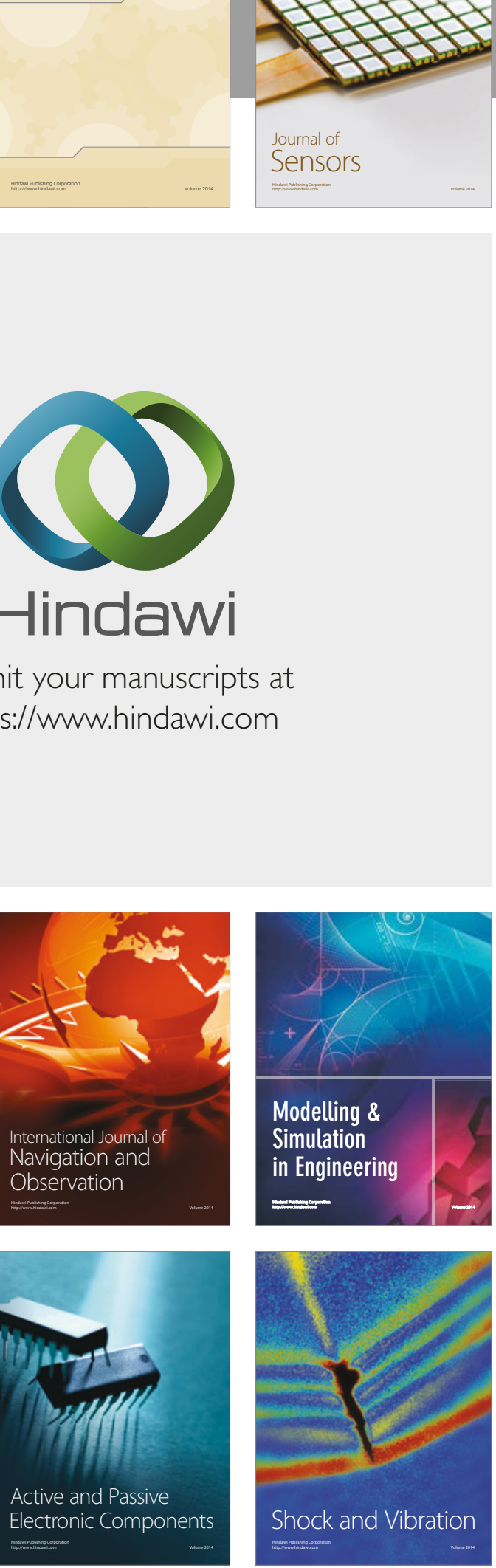
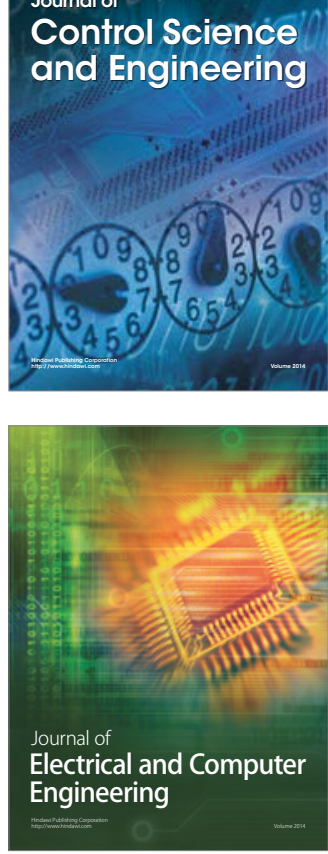

Distributed

Journal of

Control Science

and Engineering
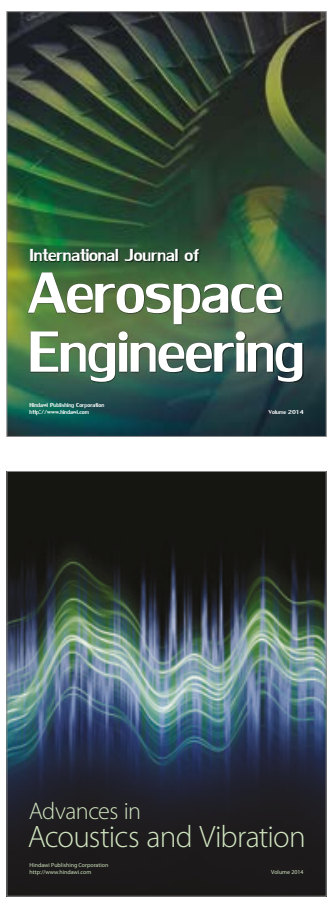

Sensor Networks 